PROCEEDINGS OF THE

AMERICAN MATHEMATICAL SOCIETY

Volume 126, Number 9, September 1998, Pages 2631-2634

S $0002-9939(98) 04355-\mathrm{X}$

\title{
THE FUGLEDE-PUTNAM THEOREM AND A GENERALIZATION OF BARRÍA'S LEMMA
}

\author{
TOSHIHIRO OKUYAMA AND KEIICHI WATANABE
}

(Communicated by Palle E. T. Jorgensen)

\begin{abstract}
Let $A$ and $B$ be bounded linear operators, and let $C$ be a partial isometry on a Hilbert space. Suppose that (1) $C A=B C$, (2) $\|A\| \geq\|B\|$, (3) $\left(C^{*} C\right) A=A\left(C^{*} C\right)$ and (4) $C\left(\|A\|^{2}-A A^{*}\right)^{1 / 2}=0$. Then we have $C A^{*}=$ $B^{*} C$.
\end{abstract}

Let $\mathcal{H}$ be a complex Hilbert space. An operator means a bounded linear operator on $\mathcal{H}$. The familiar Fuglede-Putnam theorem is stated as follows:

Theorem A (Fuglede-Putnam [3, Theorem IX.6.7]). If $A$ and $B$ are normal operators on $\mathcal{H}$ and $C$ is an operator such that $C A=B C$, then $C A^{*}=B^{*} C$.

Several authors have relaxed the normality hypothesis on $A$ and $B$ in Theorem A in various ways (for example, to hyponormality), still without restrictions on $C$, and have reached the same conclusion. However, it appears that few have attempted to place conditions on the operator $C$ in order to remove the normality hypotheses on $A$ and $B$. In this note we wish to generalize the following lemma of Barría from this point of view.

Lemma B (Barría [1, Lemma 2]). Assume that $V_{1}^{*} V_{2}=V_{2} V_{1}^{*}$, where $V_{1}$ and $V_{2}$ are isometries. Then $V_{1} V_{2}=V_{2} V_{1}$.

Now we state our result. The proof is elementary, depending on partially isometric extensions of contractions.

Theorem. Let $A$ and $B$ be bounded linear operators, and let $C$ be a partial isometry. Suppose that

(1) $C A=B C$,

(2) $\|A\| \geq\|B\|$,

(3) $\left(C^{*} C\right) A=A\left(C^{*} C\right)$ and

(4) $C\left(\|A\|^{2}-A A^{*}\right)^{1 / 2}=0$.

Then we have $C A^{*}=B^{*} C$.

Proof. We assume first that $A, B$ and $C$ are partial isometries. Then condition (4) becomes $C^{*} C \leq A A^{*}$. In particular, $C^{*} C$ and $A A^{*}$ commute. It follows from [6, Lemma 2] that $C A=B C$ is a partial isometry. Therefore, $B^{*} B$ and $C C^{*}$ commute

Received by the editors October 19, 1995 and, in revised form, January 27, 1997.

1991 Mathematics Subject Classification. Primary 47A62, 47A99; Secondary 47B20.

(C)1998 American Mathematical Society 
by [6, Lemma 2] again. Then we have

$$
\begin{aligned}
B^{*} C & =B^{*} C A A^{*}=B^{*} B C A^{*}=B^{*} B C C^{*} C A^{*} \\
& =C C^{*} B^{*} B C A^{*}=C(B C)^{*}(B C) A^{*} \\
& =C(C A)^{*}(C A) A^{*} \\
& =C A^{*} C^{*} C A A^{*}=C C^{*} C A^{*} A A^{*}=C A^{*} .
\end{aligned}
$$

Thus the theorem is true for partial isometries $A, B$ and $C$.

Now, let $A, B$ and $C$ satisfy the hypotheses of the theorem. Dividing by $\|A\|$, we may assume that $\|A\|=1$ and $\|B\| \leq 1$.

We define operator matrices $\widetilde{A}, \widetilde{B}$ and $\widetilde{C}$ by

$$
\widetilde{A}=\left[\begin{array}{cc}
A & \left(1-A A^{*}\right)^{1 / 2} \\
0 & 0
\end{array}\right], \quad \widetilde{B}=\left[\begin{array}{cc}
B & \left(1-B B^{*}\right)^{1 / 2} \\
0 & 0
\end{array}\right], \quad \widetilde{C}=\left[\begin{array}{cc}
C & 0 \\
0 & 0
\end{array}\right] .
$$

Then $\widetilde{A}, \widetilde{B}$ and $\widetilde{C}$ are partial isometries on $\mathcal{H} \oplus \mathcal{H}$ and satisfy

$$
\begin{gathered}
\widetilde{C}^{*} \widetilde{C}=\left[\begin{array}{cc}
C^{*} C & 0 \\
0 & 0
\end{array}\right] \leq\left[\begin{array}{ll}
I & 0 \\
0 & 0
\end{array}\right]=\widetilde{A} \widetilde{A}^{*}, \\
\left(\widetilde{C}^{*} \widetilde{C}\right) \widetilde{A}=\left[\begin{array}{cc}
\left(C^{*} C\right) A & \left(C^{*} C\right)\left(1-A A^{*}\right)^{1 / 2} \\
0 & 0
\end{array}\right]=\widetilde{A}\left(\widetilde{C}^{*} \widetilde{C}\right),
\end{gathered}
$$

and

$$
\widetilde{C} \widetilde{A}=\left[\begin{array}{cc}
C A & C\left(1-A A^{*}\right)^{1 / 2} \\
0 & 0
\end{array}\right]=\left[\begin{array}{cc}
B C & 0 \\
0 & 0
\end{array}\right]=\widetilde{B} \widetilde{C}
$$

Therefore, by the first paragraph of the proof, we have $\widetilde{C} \widetilde{A}^{*}=\widetilde{B}^{*} \widetilde{C}$. This implies that $C A^{*}=B^{*} C$.

Now we present an example which is not covered by the Fuglede-Putnam theorem or an existing generalization of it.

Example. Let

$$
A=\left[\begin{array}{ccccccc}
0 & 0 & a_{1} & & & & \\
& 0 & 0 & a & & & 0 \\
& & 0 & 0 & a_{2} & & \\
& & & 0 & 0 & a & \\
0 & & & & \ddots & \ddots & \ddots
\end{array}\right], \quad B=\left[\begin{array}{ccccccc}
0 & 0 & a & & & & 0 \\
& 0 & 0 & b_{1} & & & \\
& & 0 & 0 & a & & \\
& & 0 & 0 & b_{2} & \\
0 & & & \ddots & \ddots & \ddots
\end{array}\right]
$$

and

$$
C=\left[\begin{array}{ccccccc}
0 & 1 & 0 & & & & 0 \\
& 0 & 0 & 0 & & & \\
& & 0 & 1 & 0 & & \\
& & & 0 & 0 & 0 & \\
0 & & & & \ddots & \ddots & \ddots
\end{array}\right]
$$

where $\left\{a_{n}\right\}$ and $\left\{b_{n}\right\}$ are bounded sequences of complex numbers such that $a=$ $\sup _{n}\left|a_{n}\right| \geq \sup _{n}\left|b_{n}\right|$.

Then $B$ is not $M$-hyponormal [7], indeed, in general it is not even a dominant [9] or $\mathcal{Y}$-class operator. (We point out that an operator $T$ is said to be $M$-hyponormal if there exists a constant $M \geq 1$ such that $(T-\lambda)(T-\lambda)^{*} \leq M^{2}(T-\lambda)^{*}(T-\lambda)$ for any 
complex number $\lambda$. An operator $T$ is said to be dominant if for any complex number $\lambda$ there exists a number $M_{\lambda} \geq 1$ such that $(T-\lambda)(T-\lambda)^{*} \leq M_{\lambda}^{2}(T-\lambda)^{*}(T-\lambda)$.

For $\alpha>0$ an operator $T$ is said to be in $\mathcal{Y}_{\alpha}$ if there exists a number $M_{\alpha} \geq 1 \mathrm{such}$ that $\left|T^{*} T-T T^{*}\right|^{\alpha} \leq M_{\alpha}^{2}(T-\lambda)^{*}(T-\lambda)$ for any complex number $\lambda$. An operator $T$ is said to be in $\mathcal{Y}$ (or to be of $\mathcal{Y}$-class) if $T$ is in $\mathcal{Y}_{\alpha}$ for some $\alpha \geq 1$.)

Therefore, neither the Fuglede-Putnam theorem nor any existing generalizations apply. However, since $A, B$ and $C$ satisfy the hypotheses of our theorem, we can conclude that $C A^{*}=B^{*} C$.

Remark 1 . We cannot merely drop condition (3) in the Theorem. For example, let $U$ be the unilateral shift. Take $A=B=U^{*}$ and $C=\left(U^{*}\right)^{2}$. Then $A, B$ and $C$ satisfy (1), (2) and (4), but $C A^{*} \neq B^{*} C$ immediately.

Remark 2. We cannot merely drop condition (2) in the Theorem. For example, put

$$
A=\left[\begin{array}{ccccccc}
0 & 0 & 1 & & & & \\
& 0 & 0 & 1 & & & 0 \\
& & 0 & 0 & 1 & & \\
& & & 0 & 0 & 1 & \\
0 & & & & \ddots & \ddots & \ddots
\end{array}\right], \quad B=\left[\begin{array}{cccccccc}
0 & 1 & 1 & & & & & \\
& 0 & 0 & 1 & & & & 0 \\
& & 0 & 0 & 1 & & & \\
& & 0 & 0 & 1 & \\
0 & & & \ddots & \ddots & \ddots &
\end{array}\right]
$$

and

$$
C=\left[\begin{array}{cccccccc}
0 & 1 & 0 & & & & & \\
& 0 & 0 & 0 & & & & 0 \\
& & 0 & 1 & 0 & & & \\
& & & 0 & 0 & 0 & & \\
0 & & & & \ddots & \ddots & \ddots &
\end{array}\right]
$$

Then $A, B$ and $C$ satisfy (1), (3) and (4), but $C A^{*} \neq B^{*} C$.

Remark 3. We cannot merely drop condition (4) in the Theorem. For example, put

$$
A=\left[\begin{array}{ccccccc}
0 & 0 & 2 & & & & \\
& 0 & 0 & 1 & & & 0 \\
& & 0 & 0 & 1 & & \\
& & & 0 & 0 & 1 & \\
0 & & & & \ddots & \ddots & \ddots
\end{array}\right], \quad B=\left[\begin{array}{cccccccc}
0 & 0 & 1 & & & & & \\
0 & 0 & 0 & 1 & & & & 0 \\
& 1 & 0 & 0 & 1 & & & \\
& & 0 & 0 & 0 & 1 & & \\
& & & 0 & 0 & 0 & 1 & \\
0 & & & & \ddots & \ddots & \ddots & \ddots
\end{array}\right]
$$

and

$$
C=\left[\begin{array}{ccccccc}
0 & 1 & 0 & & & & 0 \\
& 0 & 0 & 0 & & & \\
& & 0 & 1 & 0 & & \\
& & & 0 & 0 & 0 & \\
0 & & & & \ddots & \ddots & \ddots
\end{array}\right]
$$

Then $A, B$ and $C$ satisfy (1), (2) and (3), but $C A^{*} \neq B^{*} C$. 


\section{REFERENCES}

1. J. Barría, The commutative product $V_{1}^{*} V_{2}=V_{2} V_{1}^{*}$ for isometries $V_{1}$ and $V_{2}$, Indiana Univ. Math. J. 28 (1979), 581-585. MR 80h:47021

2. S. K. Berberian, Note on a theorem of Fuglede and Putnam, Proc. Amer. Math. Soc. 10 (1959), 175-182. MR 21:6548

3. J. B. Conway, A Course in Functional Analysis (2nd ed.), Springer-Verlag, New York, 1990. MR 91e:46001

4. B. Fuglede, A commutativity theorem for normal operators, Proc. Nat. Acad. Sci. 36 (1950), 35-40. MR 11:371c

5. T. Furuta, On relaxation of normality in the Fuglede-Putnam theorem, Proc. Amer. Math. Soc. 77 (1979), 324-328. MR 80i:47037

6. P. R. Halmos and L. J. Wallen, Powers of partial isometries, J. Math. Mech. 19 (1970), 657-663. MR 40:4801

7. R. L. Moore, D. D. Rogers and T. T. Trent, A note on intertwining M-hyponormal operators, Proc. Amer. Math. Soc. 83 (1981), 514-516. MR 82j:47033

8. C. R. Putnam, On normal operators in Hilbert space, Amer. J. Math. 73 (1951), 357-362. MR 12:717f

9. J. G. Stampfli and B. L. Wadhwa, On dominant operators, Monatsh. Math. 84 (1977), 143153. MR 56:16428

10. K. Takahashi, On the converse of the Fuglede-Putnam theorem, Acta. Sci. Math. 43 (1981), 123-125. MR 82g:47018

11. T. Yoshino, Remark on the generalized Fuglede-Putnam theorem, Proc. Amer. Math. Soc. 95 (1985), 571-572. MR 87i:47034

Department of Mathematics, Faculty of Science, Niigata University, Niigata 950-21, JAPAN

Current address, Toshihiro Okuyama: Tsuruoka Minami Highschool, 26-31 Wakaba-cho, Tsuruoka Yamagata-ken 997-0037, Japan

E-mail address: wtnbk@scux.sc.niigata-u.ac.jp

Current address, Keiichi Watanabe: Department of Mathematics, Indiana University, Bloomington, Indiana 47405 